\title{
Application of Coiled Coil Peptides in Liposomal Anticancer Drug Delivery Using a Zebrafish Xenograft Model
}

Jian Yang, ${ }^{\dagger, \perp}$ Yasuhito Shimada, ${ }^{*},{ }^{\ddagger}, \perp$ René C. L. Olsthoorn, ${ }^{\dagger}$ B. Ewa Snaar-Jagalska, ${ }^{\dagger}$ Herman P. Spaink, ${ }^{\ddagger}$ and Alexander Kros* ${ }^{\dagger}$

${ }^{\dagger}$ Leiden Institute of Chemistry-Supramolecular and Biomaterial Chemistry, Leiden University, Einsteinweg 55, 2333CC Leiden, The Netherlands

${ }^{\ddagger}$ Institute of Biology, Leiden University, Leiden 2311 EZ, The Netherlands

${ }^{\S}$ Department of Molecular and Cellular Pharmacology, Pharmacogenomics and Pharmacoinformatics, Mie University Graduate School of Medicine, Mie 514-8507, Japan

\section{Supporting Information}

ABSTRACT: The complementary coiled coil forming peptides $\mathrm{E}_{4}\left[(\text { EIAALEK })_{4}\right]$ and $\mathrm{K}_{4}\left[(\mathrm{KIAALKE})_{4}\right]$ are known to trigger liposomal membrane fusion when tethered to lipid vesicles in the form of lipopeptides. In this study, we examined whether these coiled coil forming peptides can be used for drug delivery applications. First, we prepared $\mathrm{E}_{4}$ peptide modified liposomes containing the far-red fluorescent dye TOPRO-3 iodide ( $\mathrm{E}_{4}$-Lipo-TP3) and confirmed that $\mathrm{E}_{4}$-liposomes could deliver TP3 into HeLa cells expressing $K_{4}$ peptide on the membrane (HeLa-K) under cell culture conditions in a selective manner. Next, we prepared doxorubicin-containing $\mathrm{E}_{4}$-liposomes $\left(\mathrm{E}_{4}\right.$-Lipo-DOX) and confirmed that $\mathrm{E}_{4}$-liposomes could also deliver DOX into HeLa-K cells. Moreover, $\mathrm{E}_{4}$-Lipo-DOX showed enhanced cytotoxicity toward HeLa-K cells compared to free doxorubicin. To prove the suitability of $\mathrm{E}_{4} / \mathrm{K}_{4}$ coiled coil formation for in vivo drug delivery, we injected $\mathrm{E}_{4}$-Lipo-TP3 or $\mathrm{E}_{4}$-Lipo-DOX into zebrafish xenografts of HeLa-K. As a result, $\mathrm{E}_{4}$-liposomes delivered TP3 to the implanted HeLa-K cells, and $\mathrm{E}_{4}$-Lipo-DOX could suppress cancer proliferation in the xenograft when compared to nontargeted conditions (i.e., zebrafish xenograft with free DOX injection). These data demonstrate that coiled coil formation enables drug selectivity and efficacy in vivo. It is envisaged that these findings are a step forward toward biorthogonal targeting systems as a tool for clinical drug delivery.

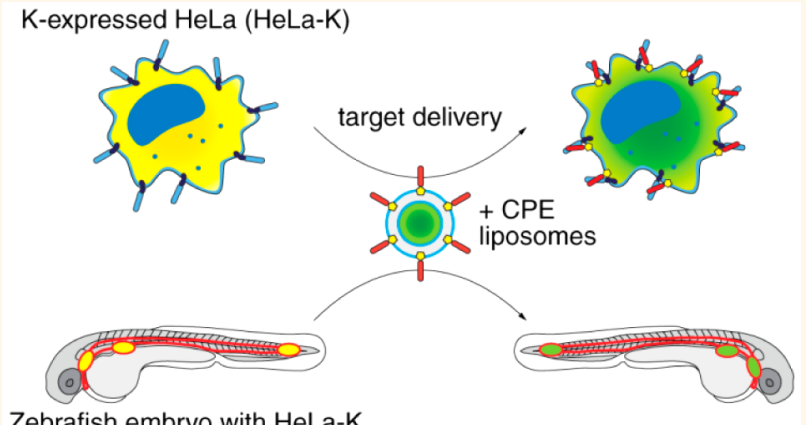

Zebrafish embryo with HeLa-K
ᄂ he design of anticancer drug delivery systems is of great interest, as many of the drugs in the clinic cause serious toxic side effects due to nonspecific cytotoxicity, outweighing the therapeutic effect. Therefore, targeted drug delivery systems are being developed, for example, micellar-, liposomal-, and nanoparticle-based drug formulations combined with peptide or surface-antibody targeting, photochemical internalization, and ultrasound stimulation. ${ }^{1}$ of these, liposomes are the most clinically established nanosystems for drug delivery, e.g., doxorubicin (DOX)-containing liposomes. ${ }^{2,3}$ However, these clinically approved drugs are nontargeted and rely on the enhanced permeability and retention effect, resulting in cardiotoxicity, thereby limiting their cumulative dose in cancer patients. To overcome this problem, liposomes have been conjugated with active targeting ligands, such as antibodies and cell-penetrating peptides, for 
Scheme 1. Drug Delivery by E4/K4 Coiled Coil Formation in Cells (A) and Zebrafish (B)

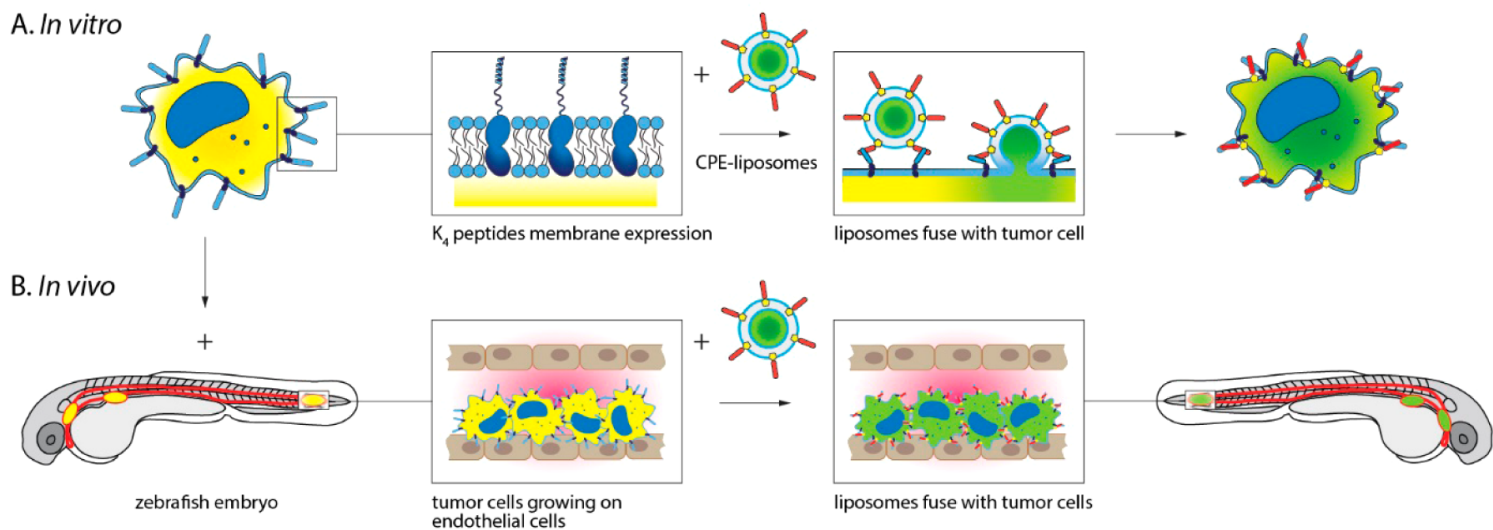

artificial biorthogonal targeting system that would be able to target liposomes and other nanoparticles more efficiently to the tissue of interest. In addition, this targeting system should also be able to enhance cellular uptake, preferentially in a nonendocytic manner. For this, synthetic coiled coils are attractive candidates as a new targeting motif. ${ }^{8,9}$ Coiled coil motifs are found in approximately $10 \%$ of all protein sequences in nature, ${ }^{10}$ and many of these noncovalent peptide motifs play a vital role in the efficient transport of molecules across membranes. For example, in the process of HIV infection, viral entry into CD4-positive cells is accomplished by intramolecular coiled coil formation between helices of viral glycoprotein gp41. ${ }^{11}$ In neuronal exocytosis, docking of transport vesicles to the target plasma membrane is mediated by the coiled coil formation of complementary SNARE protein subunits on the opposing membranes. ${ }^{12}$ This forces the opposing membranes into close proximity, resulting ultimately in lipid mixing followed by pore formation and concomitant content transfer. These natural systems have inspired researchers to design drug delivery systems based on noncovalent binding of two macromolecules. To introduce bioactive compounds into the cytosol of the target cells, various non-natural coiled coils have been used to decorate nanoparticles, ${ }^{13,14}$ which in turn are complexed to DNA antisense oligonucleotides, ${ }^{15}$ short interfering RNAs, $^{16}$ proteins, $^{17}$ drugs, ${ }^{18,19}$ or vaccines. ${ }^{20}$ Recently coiled coil motifs have also been used in drug-free therapeutic systems. ${ }^{21,22}$

In our previous study, we developed a fully synthetic membrane fusion system composed of a complementary pair ${ }^{23}$ of lipidated coiled coil peptides, denoted $\mathrm{K}$ and $\mathrm{E},{ }^{18}$ which are covalently linked to cholesterol anchors via a poly(ethylene glycol) linker, yielding lipopeptides CPK and CPE. ${ }^{24} \mathrm{We}$ demonstrated that this complementary lipopeptide pair was able to induce efficient fusion between liposomes. ${ }^{25,26}$ The E/K coiled coil formation is thought to be responsible for specific molecular recognition, ${ }^{27,28}$ and recently membrane fusion between liposomes and cells without triggering endocytosis was achieved (Yang et al., unpublished data). This system seems suitable for drug delivery, as the drug inside these liposomes avoids lysosomal degradation. While coiled coil formation induced membrane fusion has shown to be successful to deliver drugs in vitro, it has to be confirmed whether these synthetic peptide pairs are also functional in an in vivo environment. In this study we therefore used a human cancer cell xenograft in a zebrafish embryo to investigate whether coiled coil formation can be used to specifically deliver drugs to tumor cells in vivo.

\section{RESULTS AND DISCUSSION}

$E_{4}$-Liposomes Deliver TP3 to HeLa Cells That Genetically Express $\mathrm{K}_{4}$ Peptide in Vitro. Our objective is to use a pair of complementary coiled coil forming peptides, $\mathrm{E}$ and $\mathrm{K},{ }^{29}$ of which one is conjugated to a liposome to introduce chemicals into peptide-conjugated cancer cells in zebrafish (Scheme 1). The coiled coil peptide E, (EIAALEK) $)_{4}$, was conjugated to a $\mathrm{PEG}_{4}$ spacer $(\mathrm{PEG}=\operatorname{poly}($ ethylene glycol $)$ ) and cholesterol linker, yielding lipopeptide $\mathrm{CPE}_{4}$, then inserted into the bilayer of liposomes containing the fluorescent dye TO-PRO-3 iodide (TP3) (denoted $\mathrm{E}_{4}$-Lipo-TP3; Scheme 1A). TP3 is a DNA-intercalating fluorescent dye and cell membrane impermeable and stains DNA only when it is actively taken up by live cells. ${ }^{30}$ We previously confirmed that $\mathrm{E}_{4}$-Lipo-TP3 could deliver TP3 into the cytosol of HeLa cells that were pretreated with cholesterol-PEG $\mathrm{P}_{4}-\mathrm{K}_{4}\left(\mathrm{CPK}_{4}\right){ }^{28}$ While targeted drug delivery under in vitro conditions was thus successfully achieved, demonstrating the in vivo functionality of these synthetic peptides would be a significant step forward. Due to the fast turnover of membrane components in dividing cells, $\mathrm{CPK}_{4}$ inserted in a cell membrane is not expected to remain throughout the zebrafish xenotransplantation setup and experiments. For this reason, we created HeLa cells constitutively expressing a genetically encoded $\mathrm{K}_{4}$ peptide on their cell membrane (denoted HeLa-K), by fusing it to the transmembrane domain of the human platelet-derived growth factor receptor (PDGFR-TMD). ${ }^{31}$ The fusion protein is preceded by a mouse IgK-leader sequence for efficient secretion and localization on the cell membrane of the $\mathrm{K}_{4}$-fusion protein. Functional display of the $\mathrm{K}_{4}$ peptide on the outside of the cell membrane was verified in a binding experiment using a carboxyfluorescein-labeled $\mathrm{E}_{4}$ peptide (fluorescent $\mathrm{E}_{4}$; Figure S1A). Analogously to HeLa-K cells, we also created HeLa-E cells that potentially expressed $\mathrm{E}_{4}$ peptide on their cell membrane and HeLa-ctrl cells that only expressed the transmembrane domain of PDGFR. Fluorescently labeled $\mathrm{CPK}_{4}$-liposomes $\left(\mathrm{K}_{4}\right.$-Lipo-NBD) were bound to the cell membrane of HeLa-E cells (Figure S2); however fluorescent $\mathrm{E}_{4}$ peptide was taken up by the cells (Figure S1C). Ono et al. already demonstrated that the positive charge of the $\mathrm{K}_{4}$ peptide allowed nonspecific binding to negatively charged cell membranes. ${ }^{32}$ Thus, we decided to continue with HeLa-K cells and $\mathrm{E}_{4}$ peptide in the following study. 


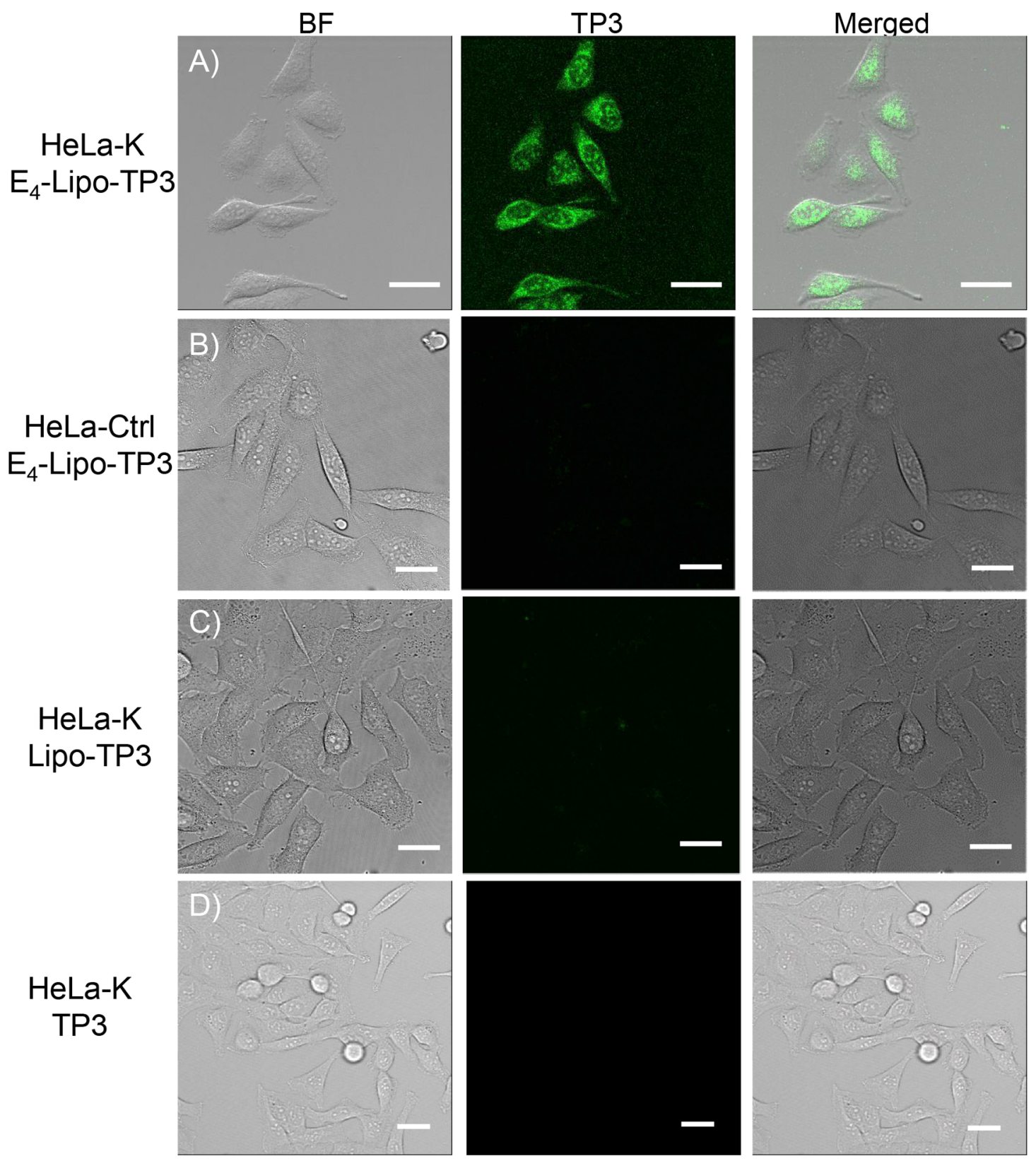

Figure 1. $\mathrm{E}_{4} / \mathrm{K}_{4}$ coiled coil formation mediated TP3 delivery into the cytosol. HeLa-K or control HeLa (HeLa-ctrl) cells were treated with 0.25 $\mathrm{mM} \mathrm{E} \mathrm{E}_{4}$-Lipo-TP3, Lipo-TP3 (without $\mathrm{E}_{4}$ peptide), or $2.5 \mu \mathrm{M}$ free TP3 for $15 \mathrm{~min}$. After three washes with culture medium, the cells were imaged. $\mathrm{E}_{4}$-Lipo-TP3 can deliver the TP3 into HeLa-K cells (A), not into HeLa-ctrl cells (B). (C) Without $\mathrm{E}_{4}$ peptide, liposomes could not deliver the TP3 into HeLa-K cells. (D) TP3 without liposome also could not deliver TP3. Green: TP3. The scale bar represents $25 \mu \mathrm{m}$.

To investigate whether $\mathrm{E}_{4}$-liposomes could deliver TP3 to HeLa-K cells, HeLa-K cells were exposed to $\mathrm{E}_{4}$-Lipo-TP3 for 15 min. This resulted in the appearance of a bright TP3 signal in the cells (Figure 1A). Control experiments in which nonmodified TP3-liposomes (Lipo-TP3; Figure 1C) or free TP3 (Figure 1D) was added to the HeLa-K cells showed less fluorescent signal in the cells. In addition, when $\mathrm{E}_{4}$-Lipo-TP3 was added to control HeLa cells (HeLa-ctrl), which express only PDGFR-TMD, also no TP3 signal was observed (Figure 1B). These results indicate that the coiled coil formation between $\mathrm{E}_{4}$ on the liposome and $\mathrm{K}_{4}$ on the cell membrane leads to delivery of liposomal contents into the cell in a selective manner.

$\mathrm{E}_{4}$-Liposomes Can Deliver TP3 in a Zebrafish Xenograft of HeLa-K Cells. Zebrafish is becoming a suitable model for characterization of nanoparticles against cancer, because of phenotypic and molecular conservation of cancer development in zebrafish and humans. ${ }^{33}$ In addition, the transparency of zebrafish embryos enables visualizing fluorescently labeled cancer cells and nanoparticles through their body wall. To monitor the fate of the implanted cells in zebrafish, HeLa-K and HeLa-ctrl cells were labeled with a red fluorescent protein that was expressed from a transfected retroviral vector expressing tdTomato (tdTom). We injected the resulting HeLa-K-tdTom or HeLa-ctrl-tdTom cells into the blood circulation of zebrafish larvae at $48 \mathrm{~h}$ postfertilization (hpf), according to a previous study. ${ }^{34}$ We used transgenic zebrafish that express vascular-specific EGFP to visualize their vasculatures. Five hours post cell injection (hpi), we confirmed that the injected cells accumulated in the caudal hematopoietic 
(A)

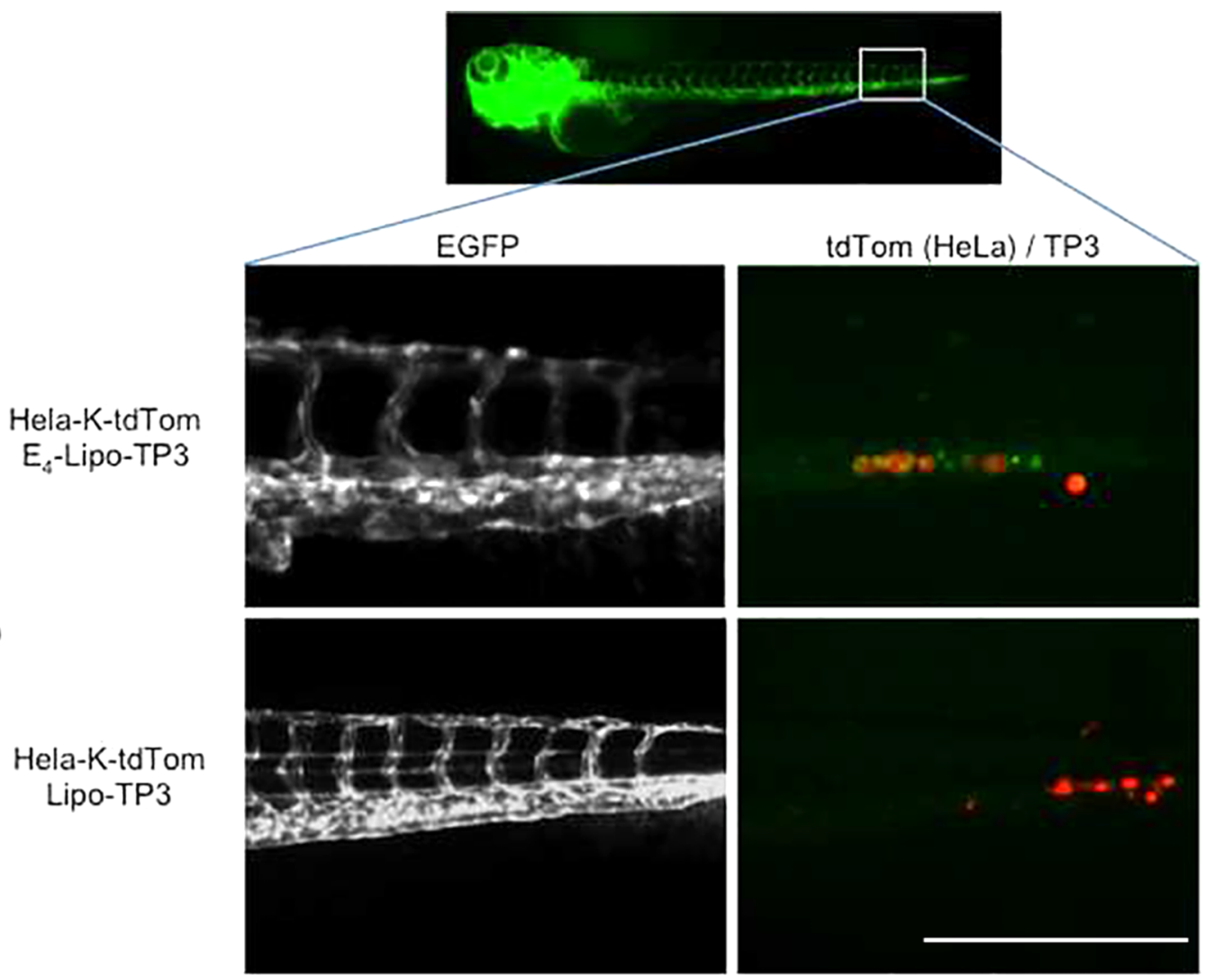

Figure $2 . \mathrm{E}_{4} / \mathrm{K}_{4}$ coiled coil formation delivers the content in the liposome to cancer cells in the xenograft zebrafish. HeLa-K cells (50-100 cells) were injected into the duct of Cuvier of $48 \mathrm{hpf}$ zebrafish. Five hours after implantation (hpi), $1 \mathrm{~nL}$ of $1 \mathrm{mM} \mathrm{E}_{4}$-Lipo-TP3 or Lipo-TP3 was injected into the CV and imaged at $72 \mathrm{hpf}$. (A) $\mathrm{E}_{4}$-Lipo-TP3 can deliver the TP3 to HeLa-K in the xenograft zebrafish, while (B) LipoTP3 could not. White, red, and green indicate vasculatures, cancer cells, and TP3, respectively. The scale bar represents $200 \mu \mathrm{m}$.

(CHT) region. Then, $\mathrm{E}_{4}$-Lipo-TP3 or Lipo-TP3 was injected into the circulation from the posterior cardinal vein $(\mathrm{CV})$. Immediately after $\mathrm{E}_{4}$-Lipo-TP3 injection, no TP3 signal could be detected in the xenografts. However, at $24 \mathrm{hpi}$, TP3 accumulation was observed in HeLa-K-tdTom cells in the CHT region (Figure 2A). Control experiments in which one of the two peptides was omitted (HeLa-K with Lipo-TP3 (Figure 2B) and free TP3 [Figure S2A], and HeLa-ctrl with $\mathrm{E}_{4}$-Lipo-TP3 [Figure S2B]) showed no TP3 uptake by the HeLa cells. These results demonstrated that $\mathrm{E}_{4} / \mathrm{K}_{4}$ coiled coil formation regulates delivery of liposomal cargo to the targeted cells in an in vivo setting.

$\mathrm{E}_{4} / \mathrm{K}_{4}$ Coiled Coil Formation Enhances DOX Cytotoxicity. Having demonstrated the feasibility of the $\mathrm{E}_{4} / \mathrm{K}_{4}$ system to target xenografted cancer cells in zebrafish, we next investigated whether our $\mathrm{E}_{4}$-liposomes could also deliver an anticancer drug such as doxorubicin ( $\mathrm{E}_{4}$-Lipo-DOX). DOX has been commonly used as a routine anticancer drug in combined chemotherapy against a variety of tumors. ${ }^{35}$ DOX interacts with DNA by intercalation and disruption of topoisomerase-IImediated DNA repair, ultimately leading to cell death. ${ }^{36}$ Noteworthy, DOX is a popular research tool due to its inherent fluorescence associated with the central anthracycline chromophore group. This allows visualization of DOX distribution in various tissues or cells via fluorescence imaging. ${ }^{37}$ Indeed, we were able to visualize DOX intake into HeLa-K cells. After 15 min incubation with $0.25 \mathrm{mM} \mathrm{E} \mathrm{E}_{4}$-Lipo-DOX (1 mM DOXcontaining $\mathrm{E}_{4}$-liposome, equal to $0.25 \mathrm{mM}$ free-DOX concentration), DOX fluorescent signal was already observed in the cytosol (Figure 3A). Five hours after washout of $\mathrm{E}_{4}$-LipoDOX from the culture medium, DOX signal had increased (Figure 3B). Mohan et al. reported that the DOX fluorescence

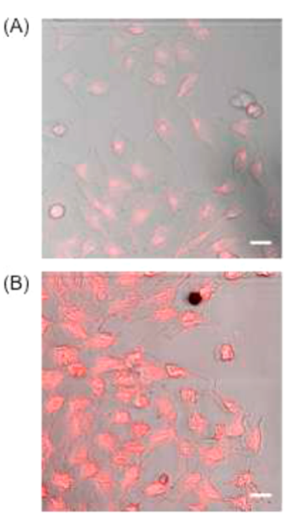

(C)

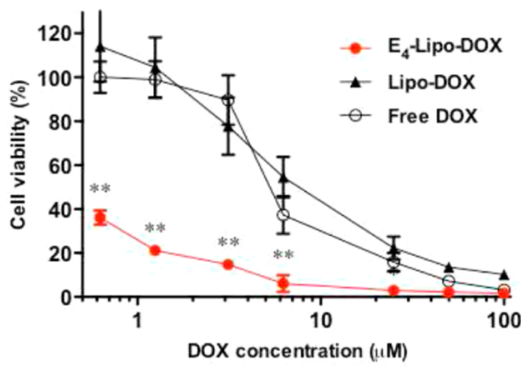

Figure 3. $E_{4} / K_{4}$ coiled coil formation promotes doxorubicin intake and cytotoxicity. HeLa- $\mathrm{K}$ cells were incubated with $0.25 \mathrm{mM} \mathrm{E}_{4^{-}}$ Lipo-DOX for $15 \mathrm{~min}$, and after three washes with culture medium, the cells were imaged (A). After $5 \mathrm{~h}$, the cells were imaged again using the same settings (B). Red indicates DOX. The scale bar represents $25 \mu \mathrm{m}$. For images of negative controls, see supplementary Figure S3. (C) Cell viability after DOX delivery. HeLa-K cells were incubated with different concentrations of $E_{4}$ Lipo-DOX (red circles), Lipo-DOX (black triangles), or free-DOX (open circles) for $12 \mathrm{~h}$, then washed three times. Twenty-four hours after treatment, cell viability was measured. $n=4$, error bar indicates SD. $* P<0.05$. 
(A)
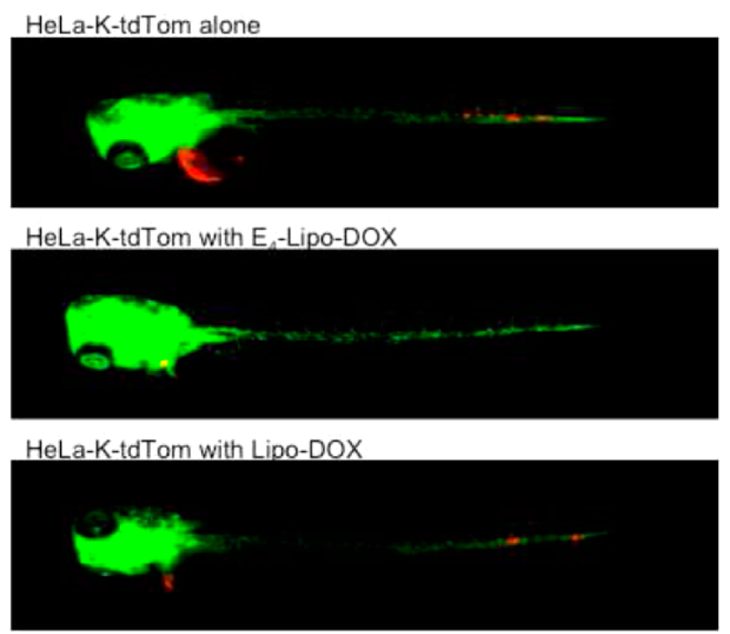

HeLa-K-tdTom with free DOX

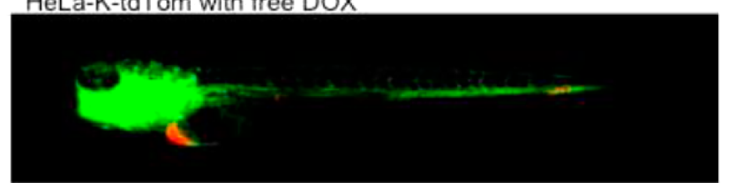

(B)

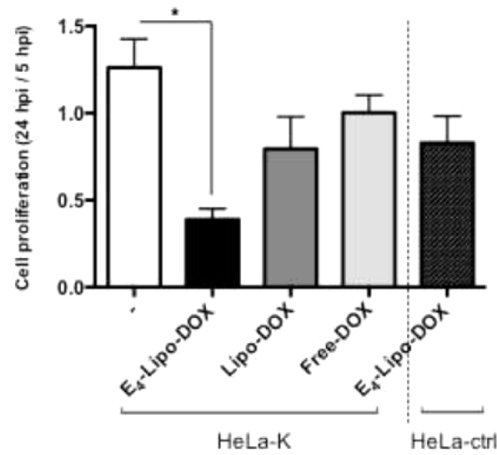

Figure 4. $\mathrm{E}_{4} / \mathrm{K}_{4}$ coiled coil formation enhances anticancer property of doxorubicin in the xenografts. (A) HeLa-K or HeLa-ctrl cells were injected into the duct of Cuvier of $48 \mathrm{hpf}$ zebrafish. After $5 \mathrm{~h}, 1 \mathrm{~nL}$ of $1 \mathrm{mM} \mathrm{E}$-Lipo-DOX, Lipo-DOX, or $0.25 \mathrm{mM}$ free-DOX was injected from the CV and imaged at $72 \mathrm{hpf}$. HeLa-K xenograft with $\mathrm{E}_{4}$-Lipo-DOX injection (left upper panel) shows the decrease of the injected cells. Green and red indicate vasculatures and cancer cells, respectively. (B) Quantification of the cancer proliferation in the xenografts. Cancer cell proliferation was calculated as the ratio of tdTomato fluorescence intensity in the tumor area relative to that at 5 hpi. $n=10-12$, error bar $=$ $+\mathrm{SE}, * P<0.05$.

inside cells increases in a time-dependent manner because of the alternation of DOX's binding partners: DNA, histones, and phospholipids. ${ }^{37}$ Thus, we assumed that DOX-phospholipid and DOX-DNA-histone complexes would be detected at this time point. In contrast, control experiments in which $\mathrm{E}_{4}$ peptide was omitted from the liposomes (HeLa-K with Lipo-DOX [Figure S3A] or using free DOX [Figure S3B]) showed very low cellular uptake of DOX. As expected, free DOX was not taken up by HeLa-ctrl (Figure S3C) and HeLa-E cells (Figure S3D), similarly to HeLa-K. We repeated the experiment in the presence of several well-known endocytosis inhibitors in order to study the pathway of cellular uptake (Figure S6). It was shown that uptake via endocytosis was the minor pathway, suggesting that fusion might be the major pathway. However, additional studies are required to confirm this finding.

To investigate whether coiled coil mediated delivery enhances the cytotoxicity of DOX, we treated HeLa-K cells with $\mathrm{E}_{4}$-Lipo-DOX, Lipo-DOX, or free DOX for $12 \mathrm{~h}$. As a result, $1 \mu \mathrm{M} \mathrm{E}_{4}$-Lipo-DOX exhibited around $80 \%$ reduction in the number of HeLa-K cells, while free DOX had no measurable effect at the same concentration (Figure 3C). In addition, Lipo-DOX showed the same toxicity as that of free DOX to HeLa-K cells. This result indicates that $\mathrm{E}_{4} / \mathrm{K}_{4}$ coiled coil formation increases the cytotoxicity of DOX and reduces the dose required for the induction of cancer cell death. Interestingly, under nontargeting conditions with free DOX, HeLa-ctrl cells (Figure S4, open circles) seem more sensitive than HeLa-K cells (Figure 3C, open circles). Membranous expression of $\mathrm{K}_{4}$ peptide might have some protective effects to the cells; however the $\mathrm{IC}_{50}$ values of $\mathrm{DOX}$ for HeLa-ctrl and HeLa-K cells are similar: 4.0 and $5.3 \mu \mathrm{M}$, respectively.
$\mathrm{E}_{4} / \mathrm{K}_{4}$ Coiled Coil Formation Enhances Anticancer Efficacy of DOX in Xenografts. To investigate the toxicity of DOX toward cancer cells in vivo, we conducted another zebrafish xenograft experiment similar to the TP3 study above. We injected HeLa-K-tdTom cells into the circulation from the duct of Cuvier at $48 \mathrm{hpf}$, followed by $\mathrm{E}_{4}$-Lipo-DOX, Lipo-DOX, or free DOX injection at $5 \mathrm{hpi}$. Twenty-four hours after DOX injection, these xenografts were imaged using fluorescent microscopy (Figure 4A). As illustrated by the images in Figure $4 \mathrm{~A}$, the fluorescence of the Hela-K-tdTom cells was substantially more reduced by treatment with $\mathrm{E}_{4}$-Lipo-DOX (second image) than by treatment with either Lipo-DOX (third image) or free DOX (fourth image). Quantification of the fluorescent intensities showed that injection of $\mathrm{E}_{4}$-Lipo-DOX resulted in a significantly $(P<0.05)$ reduced tumor cell proliferation compared to Lipo-DOX or free DOX treatment (Figure 4B). Other nontargeting conditions (HeLa-K-tdTom with Lipo-DOX and free-DOX) did not exhibit significant reduction of cancer cell burden, similarly to the in vitro experiment in Figure 3C. As described in the Materials and Methods, we injected $1 \mathrm{~nL}$ of $1 \mathrm{mM}$ liposomes containing 0.25 mM DOX. Based on a wet weight estimation of $240 \mu \mathrm{g}$ for zebrafish larvae, ${ }^{38}$ the injection volume of DOX was equal to 1 $\mu \mathrm{mol} / \mathrm{kgBW}$ (kilogram per body weight). In the clinic, DOX is usually injected into the circulation of cancer patients at a concentration of $2.5 \mathrm{mg} / \mathrm{kgBW}$, which equals $5.16 \mu \mathrm{mol} /$ kgBW. ${ }^{39}$ Therefore, the administered amount of DOX using $\mathrm{E}_{4}$ liposomes was 5-fold lower than that used in a clinical setting with lesser opportunity for exhibition of cardiac toxicity, while the anticancer efficacy of $\mathrm{E}_{4}$-Lipo-DOX is far greater than that of free-DOX. 


\section{CONCLUSION}

Targeted drug delivery systems should increase the efficacy of a drug and concomitantly reduce the toxic side effects caused by off-target reactions. Coiled coil motifs were recently used in a "drug-free therapeutic system" to specifically kill cancer cells. ${ }^{40}$ In this study, we demonstrated that coiled coil formation between liposomes and live cells enables the in vivo delivery of an anticancer drug encapsulated in CPE-decorated liposomes to the targeted cells in an animal model. This is one of the first examples using a synthetic coiled coil motif for in vivo targeted drug delivery. Although this system currently requires $\mathrm{K}_{4}$ peptide expression on the cell membrane of targeted cells in zebrafish xenografts, this method can be used for animal testing of a wide range of drug candidates because of the surprising degree of functional conservation in basic cell-biological processes between zebrafish and mammals. ${ }^{41}$ Furthermore, the combination of coiled coil mediated delivery and zebrafish xenografts of human cancer has the potential to become a powerful and rapid in vivo drug-screening platform. For future clinical applications, however, it will be necessary to introduce $\mathrm{K}_{4}$ peptide to the targeted cells or tissues of interest (e.g., tumor). This might be achieved by injection of a PEGylated CPK that can be locally deshielded by irradation with light, ${ }^{27}$ conjugation of $\mathrm{K}_{4}$ peptide to cancer-specific cell membrane antibodies (e.g., Her2 antibody $^{42}$ ) or direct introduction of our $\mathrm{K}_{4}$-PDGFR-TMD construct using adeno-associated virus vector injection. ${ }^{43}$ The proposed improvements of the current method should enable spatiotemporal control of liposomal drug targeting in the near future. These approaches to introduce $\mathrm{K}_{4}$ peptide into diseased tissues or cells combined with the enhanced drug selectivity circumventing lysosomal degradation might increase the drug efficacy and reduce toxic side effects.

\section{MATERIALS AND METHODS}

Zebrafish Strain, Husbandry, and Egg Collection. Tg (fli1: EGFP) zebrafish ${ }^{44}$ were used in this study. Livestock were maintained and handled according to the guidelines from http://zfin.org. Fertilization was performed by natural spawning at the beginning of the light period, and eggs were raised at $28{ }^{\circ} \mathrm{C}$. All experimental procedures were conducted in compliance with the directives of the animal welfare committee of Leiden University.

Plasmids. The insert designs for membrane expression of $\mathrm{K}_{4}$ and $\mathrm{E}_{4}$ peptide are described in Supplementary Methods. The insert sequences were synthesized by BaseClear (Leiden, The Netherlands), then digested with SalI and NotI and ligated into episomal plasmid pEBMulti-Hyg (Wako Pure Chemicals, Osaka, Japan) to create pEBM-K and pEBM-E. The backbone vector harbors OriP (replication origin) and EBNA-1 derived from Epstein-Barr virus, which allows the distribution of this plasmid to daughter cells by episomal replication to generate stable transformants. ${ }^{45}$

Cells. HeLa human cervical cancer cells were obtained from the American Type Culture Collection (Manassas, VA, USA). The cells were maintained in Dulbecco's modified Eagle's medium (DMEM; Life Technologies, Carlsbad, CA, USA), supplemented with $10 \%$ fetal bovine serum (FCS; Life Technologies), 100 units $/ \mathrm{mL}$ of penicillin (Sigma-Aldrich, St. Louis, MO, USA), and $100 \mu \mathrm{g} / \mathrm{mL}$ streptomycin (Sigma-Aldrich) at $37{ }^{\circ} \mathrm{C}$ with $5 \% \mathrm{CO}_{2}$. To construct HeLa-K and HeLa-E cells, HeLa cells were transfected with pEBM-K and pEBM-E plasmids, respectively, and cultured in the presence of hygromycin $B$ (200 $\mu \mathrm{g} / \mathrm{mL}$; Sigma-Aldrich) for at least 2 weeks to obtain stable transformants. To create control cells (HeLa-ctrl), HeLa cells were transfected with pEBM harboring PDGFR-TMD (see Supplementary Methods). For tdTomato fluorescent protein expression, the cells were infected with tdTomato lentivirus (pLenti-tdTom-Bla; a gift from Dr. Maciej Olszewski) according to the standard protocol. The tdTomato cells were cultured in the presence of blasticidin $(10 \mu \mathrm{g} / \mathrm{mL}$; Life Technologies) to obtain stable transformants.

Cell Imaging. The cells were seeded in an eight-well slide ( $\mu$-Slide 8 well; Ibidi, Munich, Germany) at a density of $2.5 \times 10^{4}$ cells per well in DMEM-10\% FCS medium without phenol red and cultured overnight. Then, the medium was refreshed and test compounds were added (TP3 or DOX-containing liposomes, which were diluted to a final concentration using DMEM [- phenol red)]. After incubation for $15 \mathrm{~min}$, cells were washed three times with medium. The fluorescent images were acquired using a Leica TCS SP8 confocal laser scanning microscope (Leica Microsystems, Wetzlar, Germany) and merged with Leica application suite advanced fluorescence software (Leica Microsystems) or ImageJ software (National Institutes of Health, Bethesda, MD, USA). The wavelength settings for TP3 and DOX were Ex/Em: 641/662 nm (Ex laser: $633 \mathrm{~nm}$ ) and 480/580 nm (Ex laser: $532 \mathrm{~nm}$ ), respectively. ${ }^{46}$

Zebrafish Xenografts and Compound Treatment. HeLa cell implantation was performed as previously reported. ${ }^{34}$ In brief, dechorionized $48 \mathrm{hpf}$ zebrafish were anaesthetized with $0.003 \%$ tricaine (MS222; Sigma-Aldrich). Then trypsinized HeLa cells (100200 cells) were injected into the duct of Cuvier by using a Pneumatic Pico pump and a manipulator (World Precision Instruments, Sarasota, FL, USA). After implantation, zebrafish were maintained at $34{ }^{\circ} \mathrm{C}$. Five hours after implantation, $1 \mathrm{~nL}$ of test compounds was injected into the caudal vein using the Pico pump with a fine glass needle. For live imaging, xenografts were anaesthetized and mounted in $0.6 \%$ lowmelting agarose. Fluorescent image acquisition was performed using a Leica MZ16FA stereo-microscope (Leica Microsystems). Images were adjusted for brightness and contrast using ImageJ.

\section{ASSOCIATED CONTENT}

\section{S Supporting Information}

The Supporting Information is available free of charge on the ACS Publications website at DOI: 10.1021/acsnano.6b01410.

Peptide synthesis, liposome preparation, Dox release; cell assays, Dox delivery (PDF)

\section{AUTHOR INFORMATION}

\section{Corresponding Authors}

*E-mail (Y. Shimada): cobo2@doc.medic.mie-u.ac.jp.

*E-mail (A. Kros): a.kros@chem.leidenuniv.nl.

\section{Author Contributions}

${ }^{\perp}$ J. Yang and Y. Shimada contributed equally to this work.

\section{Notes}

The authors declare no competing financial interest.

\section{ACKNOWLEDGMENTS}

We are grateful to Dr. Maciej Olszewski for providing the pLenti-tdTom-Bla construct and Arwin Groenewoud for preparation of the lentivirus. J.Y. was supported by a grant from the Chinese Scholarship Council. A.K. acknowledges the support of NWO via a VICI grant (724.014.001).

\section{REFERENCES}

(1) Yu, Z.; Yan, B.; Gao, L.; Dong, C.; Zhong, J.; M, D. O.; Nguyen, B.; Hu, X.; Liang, F. Targeted Delivery of Bleomycin: a Comprehensive Anticancer Review. Curr. Cancer Drug Targets 2016, $16,509-521$.

(2) Blume, G.; Cevc, G. Liposomes for the Sustained Drug Release In vivo. Biochim. Biophys. Acta, Biomembr. 1990, 1029, 91-97.

(3) Torchilin, V. P. Recent advances with Liposomes as Pharmaceutical Carriers. Nat. Rev. Drug Discovery 2005, 4, 145-160.

(4) Brock, R. The Uptake of Arginine-Rich Cell-Penetrating Peptides: Putting the Puzzle Together. Bioconjugate Chem. 2014, 25, 863-868. 
(5) Karagiannis, E. D.; Urbanska, A. M.; Sahay, G.; Pelet, J. M.; Jhunjhunwala, S.; Langer, R.; Anderson, D. G. Rational Design of a Biomimetic Cell Penetrating Peptide Library. ACS Nano 2013, 7, $8616-8626$.

(6) Scott, R. C.; Wang, B.; Nallamothu, R.; Pattillo, C. B.; Perez-Liz, G.; Issekutz, A.; Del Valle, L.; Wood, G. C.; Kiani, M. F. Targeted Delivery of Antibody Conjugated Liposomal Drug Carriers to Rat Myocardial Infarction. Biotechnol. Bioeng. 2007, 96, 795-802.

(7) Bareford, L. M.; Swaan, P. W. Endocytic Mechanisms for Targeted Drug Delivery. Adv. Drug Delivery Rev. 2007, 59, 748-758.

(8) Diss, M. L.; Kennan, A. J. Heterotrimeric Coiled Coils with Core Residue Urea Side Chains. J. Org. Chem. 2008, 73, 9752-9755.

(9) Vandermeulen, G. W. M.; Tziatzios, C.; Klok, H. A. Reversible Self-Organization of Poly(ethylene glycol)-Based Hybrid Block Copolymers Mediated by a De Novo Four-Stranded $\alpha$-Helical Coiled Coil Motif. Macromolecules 2003, 36 (11), 4107-4114.

(10) Kohn, W. D.; Mant, C. T.; Hodges, R. S. Alpha-Helical Protein Assembly Motifs. J. Biol. Chem. 1997, 272, 2583-2586.

(11) Lawless, M. K.; Barney, S.; Guthrie, K. I.; Bucy, T. B.; Petteway, S. R., Jr.; Merutka, G. HIV-1 Membrane Fusion Mechanism: Structural Studies of the Interactions between Biologically-Active Peptides from Gp41. Biochemistry 1996, 35, 13697-13708.

(12) Clague, M. J. Membrane Transport: Take Your Fusion Partners. Curr. Biol. 1999, 9, R258-260.

(13) Martelli, G.; Zope, H. R.; Capell, M. B.; Kros, A. Coiled-Coil Peptide Motifs as Thermoresponsive Valves for Mesoporous Silica Nanoparticles. Chem. Commun. (Cambridge, U. K.) 2013, 49, 99329934

(14) Assal, Y.; Mizuguchi, Y.; Mie, M.; Kobatake, E. Growth Factor Tethering to Protein Nanoparticles via Coiled-Coil Formation for Targeted Drug Delivery. Bioconjugate Chem. 2015, 26, 1672-1677.

(15) Petrilli, R.; Eloy, J. O.; Marchetti, J. M.; Lopez, R. F.; Lee, R. J. Targeted Lipid Nanoparticles for Antisense Oligonucleotide Delivery. Curr. Pharm. Biotechnol. 2014, 15, 847-855.

(16) Varshosaz, J.; Farzan, M. Nanoparticles for Targeted Delivery of Therapeutics and Small Interfering RNAs in Hepatocellular Carcinoma. World J. Gastroenterol. 2015, 21, 12022-12041.

(17) Pechar, M.; Pola, R.; Laga, R.; Ulbrich, K.; Bednarova, L.; Malon, P.; Sieglova, I.; Kral, V.; Fabry, M.; Vanek, O. Coiled Coil Peptides as Universal Linkers for the Attachment of Recombinant Proteins to Polymer Therapeutics. Biomacromolecules 2011, 12, 36453655 .

(18) Baran, E. T.; Ozer, N.; Hasirci, V. In vivo Half Life of Nanoencapsulated L-Asparaginase. J. Mater. Sci.: Mater. Med. 2002, 13, $1113-1121$

(19) LaVan, D. A.; McGuire, T.; Langer, R. Small-Scale Systems for in vivo Drug Delivery. Nat. Biotechnol. 2003, 21, 1184-1191.

(20) Gregory, A. E.; Titball, R.; Williamson, D. Vaccine Delivery using Nanoparticles. Front. Cell. Infect. Microbiol. 2013, 3, 13.

(21) Kverka, M.; Hartley, J. M.; Chu, T. W.; Yang, J.; Heidchen, R.; Kopecek, J. Immunogenicity of Coiled-Coil Based Drug-Free Macromolecular Therapeutics. Biomaterials 2014, 35, 5886-5896.

(22) Zhang, R.; Yang, J.; Chu, T. W.; Hartley, J. M.; Kopecek, J. Multimodality Imaging of Coiled-Coil Mediated Self-Assembly in a "Drug-Free" Therapeutic System. Adv. Healthcare Mater. 2015, 4, 1054-1065.

(23) Robson Marsden, H.; Elbers, N. A.; Bomans, P. H.; Sommerdijk, N. A.; Kros, A. A Reduced SNARE Model for Membrane Fusion. Angew. Chem., Int. Ed. 2009, 48, 2330-2333.

(24) Versluis, F.; Voskuhl, J.; van Kolck, B.; Zope, H.; Bremmer, M.; Albregtse, T.; Kros, A. In situ Modification of Plain Liposomes with Lipidated Coiled Coil Forming Peptides Induces Membrane Fusion. J. Am. Chem. Soc. 2013, 135, 8057-8062.

(25) Zheng, T.; Voskuhl, J.; Versluis, F.; Zope, H. R.; Tomatsu, I.; Marsden, H. R.; Kros, A. Controlling The Rate of Coiled Coil Driven Membrane Fusion. Chem. Commun. (Cambridge, U. K.) 2013, 49, $3649-3651$.

(26) Kong, L.; Askes, S. H.; Bonnet, S.; Kros, A.; Campbell, F. Temporal Control of Membrane Fusion through Photolabile
PEGylation of Liposome Membranes. Angew. Chem., Int. Ed. 2016, $55,1396-1400$

(27) Zope, H. R.; Versluis, F.; Ordas, A.; Voskuhl, J.; Spaink, H. P.; Kros, A. In vitro and in vivo Supramolecular Modification of Biomembranes using a Lipidated Coiled-Coil Motif. Angew. Chem., Int. Ed. 2013, 52, 14247-14251.

(28) Oude Blenke, E.; van den Dikkenberg, J.; van Kolck, B.; Kros, A.; Mastrobattista, E. Coiled coil interactions for the targeting of liposomes for nucleic acid delivery. Nanoscale 2016, 8, 8955-8965.

(29) Marsden, H. R.; Korobko, A. V.; van Leeuwen, E. N.; Pouget, E. M.; Veen, S. J.; Sommerdijk, N. A.; Kros, A. Noncovalent Triblock Copolymers Based on a Coiled-Coil Peptide Motif. J. Am. Chem. Soc. 2008, 130, 9386-9393.

(30) Van Hooijdonk, C. A.; Glade, C. P.; Van Erp, P. E. TO-PRO-3 iodide: A Novel HeNe Laser-Excitable DNA Stain as an Alternative for Propidium Iodide in Multiparameter Flow Cytometry. Cytometry 1994, 17, 185-189.

(31) Szent-Gyorgyi, C.; Schmidt, B. F.; Creeger, Y.; Fisher, G. W.; Zakel, K. L.; Adler, S.; Fitzpatrick, J. A.; Woolford, C. A.; Yan, Q.; Vasilev, K. V.; Berget, P. B.; Bruchez, M. P.; Jarvik, J. W.; Waggoner, A. Fluorogen-Activating Single-Chain Antibodies for Imaging Cell Surface Proteins. Nat. Biotechnol. 2008, 26, 235-240.

(32) Ono, S.; Yano, Y.; Matsuzaki, K. Improvement of Probe Peptides for Coiled-Coil Labeling by Introducing Phosphoserines. Biopolymers 2012, 98, 234-238.

(33) Evensen, L.; Johansen, P. L.; Koster, G.; Zhu, K.; Herfindal, L.; Speth, M.; Fenaroli, F.; Hildahl, J.; Bagherifam, S.; Tulotta, C.; Prasmickaite, L.; Maelandsmo, G. M.; Snaar-Jagalska, E.; Griffiths, G. Zebrafish as a Model System for Characterization of Nanoparticles against Cancer. Nanoscale 2015, 8, 862-877.

(34) He, S.; Lamers, G. E.; Beenakker, J. W.; Cui, C.; Ghotra, V. P.; Danen, E. H.; Meijer, A. H.; Spaink, H. P.; Snaar-Jagalska, B. E. Neutrophil-Mediated Experimental Metastasis Is Enhanced by VEGFR Inhibition In a Zebrafish Xenograft Model. J. Pathol. 2012, 227, 431445.

(35) Fritze, A.; Hens, F.; Kimpfler, A.; Schubert, R.; Peschka-Suss, R Remote Loading of Doxorubicin into Liposomes Driven by a Transmembrane Phosphate Gradient. Biochim. Biophys. Acta, Biomembr. 2006, 1758, 1633-1640.

(36) Momparler, R. L.; Karon, M.; Siegel, S. E.; Avila, F. Effect of Adriamycin on DNA, RNA, and Protein Synthesis in Cell-Free Systems and Intact Cells. Cancer Res. 1976, 36, 2891-2895.

(37) Mohan, P.; Rapoport, N. Doxorubicin As a Molecular Nanotheranostic Agent: Effect of Doxorubicin Encapsulation in Micelles or Nanoemulsions on the Ultrasound-Mediated Intracellular Delivery and Nuclear Trafficking. Mol. Pharmaceutics 2010, 7, 19591973.

(38) Hagedorn, M.; Kleinhans, F. W.; Freitas, R.; Liu, J.; Hsu, E. W.; Wildt, D. E.; Rall, W. F. Water Distribution and Permeability of Zebrafish Embryos, Brachydanio Rerio. J. Exp. Zool. 1997, 278, 356371.

(39) Viswanatha Swamy, A. H.; Wangikar, U.; Koti, B. C.; Thippeswamy, A. H.; Ronad, P. M.; Manjula, D. V. Cardioprotective Effect of Ascorbic Acid on Doxorubicin-Induced Myocardial Toxicity in Rats. Indian J. Pharmacol. 2011, 43, 507-511.

(40) Wu, K.; Yang, J.; Liu, J.; Kopecek, J. Coiled-Coil Based DrugFree Macromolecular Therapeutics: in vivo efficacy. J. Controlled Release 2012, 157, 126-131.

(41) Lieschke, G. J.; Currie, P. D. Animal Models of Human Disease: Zebrafish Swim into View. Nat. Rev. Genet. 2007, 8, 353-367.

(42) Calce, E.; Monfregola, L.; Saviano, M.; De Luca, S. HER2Mediated Anticancer Drug Delivery: Strategies to Prepare Targeting Ligands Highly Specific for the Receptor. Curr. Med. Chem. 2015, 22, $2525-2538$

(43) Manfredsson, F. P. Introduction to Viral Vectors and Other Delivery Methods for Gene Therapy of the Nervous System. Methods Mol. Biol. (N. Y., NY, U. S.) 2016, 1382, 3-18. 
(44) Lawson, N. D.; Weinstein, B. M. In vivo Imaging of Embryonic Vascular Development using Transgenic zebrafish. Dev. Biol. (Amsterdam, Neth.) 2002, 248, 307-318.

(45) Shibata, M. A.; Miwa, Y.; Morimoto, J.; Otsuki, Y. Easy Stable Transfection of a Human Cancer Cell Line by Electrogene Transfer with an Epstein-Barr Virus-Based Plasmid Vector. Med. Mol. Morphol. 2007, 40, 103-107.

(46) Luo, J.; Mohammed, I.; Warmlander, S. K.; Hiruma, Y.; Graslund, A.; Abrahams, J. P. Endogenous Polyamines Reduce the Toxicity of Soluble Abeta Peptide Aggregates Associated with Alzheimer's Disease. Biomacromolecules 2014, 15, 1985-1991. 